\title{
A Review Paper on Numerical Simulation of Moving Heat Source
}

\author{
Sudesh B. Powar ${ }^{*}$, Prashant M. Patane ${ }^{\dagger}$ and Shailesh L Deshmukh ${ }^{\dagger}$ \\ †Department of Mechanical Engineering, MIT Academy of Engineering, Alandi, Pune, India \\ Accepted 02 March 2016, Available online 15 March 2016, Special Issue-4 (March 2016)
}

\begin{abstract}
The problems of heat conduction resulting from a moving heat source applies to many fields of engineering such as welding, surface hardening, moving friction between mechanical parts, laser treatments etc. Heat source models are used to define the heat flux distribution in the domain.An attempt has been made to gather the information about moving heat source problems and different heat source models. The optimal design reduces failure rates, improves product life cycle and important to all it will find out defects due to temperature distribution within the material. The analytical solutions are available for simple problems but as the complexicity increases the analytical solutions fail to predict results. Numerical method is a key to solve many complicated mathematical problems.
\end{abstract}

Keywords: Moving Heat Source, Heat Source Models.

\section{Introduction}

Stationary and moving plane heat source analysis have application in several manufacturing processes such as metal cutting, spot welding, laser cutting/surface treatment (using $\mathrm{CO} 2$ or Argon lasers) as well as tribological applications including ball bearing and gear design. Temperature profile and the rate of cooling at and near the surface can affect the metallurgical microstructures, thermal shrinkage, thermal cracking, hardness distribution, residual stresses and heat affected zones of the material. Analytical and numerical models for the prediction of the thermal fields induced by the stationary or moving heat sources are useful tools for studying the above mentioned problems. Knowing temperature distribution in tribological applications due to frictional heat generation is required to minimize thermal related problems such as lubricant break down. Generated heat at the surface of one body or at the contact interface between two bodies in industrial processes, such as laser welding, breaking systems, friction between two mediums and so on, is often modeled by using the concept of moving heat source in stationary and transient case. Most of this heat is expended on the increase in the temperature of the contact interface. This local temperature increase can strongly affect the surface properties of materials. Thus, the temperature level plays an important role in various applications and should be carefully controlled.

The various investigations can be divided into two categories:
1) Analytical solutions which are possible in some special cases of geometry and boundary conditions.

2) Numerical methods that provide results for practically any combination of geometry and boundary conditions.

There are mainly four data required in the thermal analysis of moving heat source problem

(1) The strength and distribution of the heat source.

(2) The convection of cooling media, which reflects the effect of coolant,

(3) The thermal properties of the work material, and

(4) The moving speed of the heat source.

\section{Literature Review}

A good understanding of the heat transfer process in the moving heat source can be helpful for predicting temperature distribution in workpiece. Several papers have been written on the moving heat source, some of them were seeking for analytical solution while other uses numerical methods to solve the problem. Their research has been made for particular application of moving heat source like welding, laser, grinding, etc. only.

N. Bianco, et al. A laser source with Gaussian distribution is considered moving with const velocity along motion direction as shown in Fig. 1. These 3dimensional transient conductive fields are solved by COMSOL Multiphysics code. The thermophysical properties of the material are assumed to be temperature dependent except density. They produced 
their results in two cases. a) An infinite workpiece along the motion direction with heat transfer coefficient on upper and bottom surface. b) A semiinfinite workpiece along the motion direction with negligible convective and radiative heat losses. Temperature profile and fields shows that a quasi stead state is reached. They use uniform grid structure if mesh nodes are doubled then the temperature difference of the field is less than $0.1 \%$.

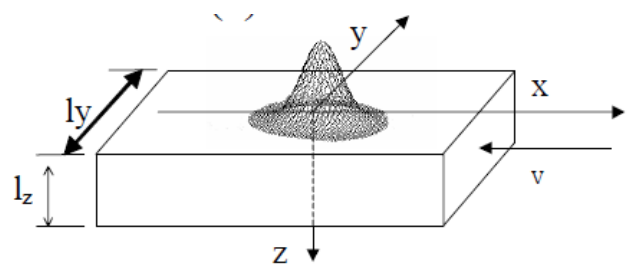

Fig.1 Sketch of workpiece with heat source distribution

A.G.Mamalis, et al. They studied finite element thermal analysis of oxide grinding wheel with implicit finite element code MARC. From the numerical results obtained from analysis it is possible to calculate maximum temperature, the distribution of temperature fields during grinding. A finite element model based on Jager's model is proposed for the simulation of grinding process. On the top surface of the workpiece heat is entering in the form of heat flux that moves along the surface. Cooling is silmulated by means of convective boundary conditions. All other sides of workpiece are considered to be adiabatic. The mesh is denser towards the grinding surface that is thermally loaded surface.

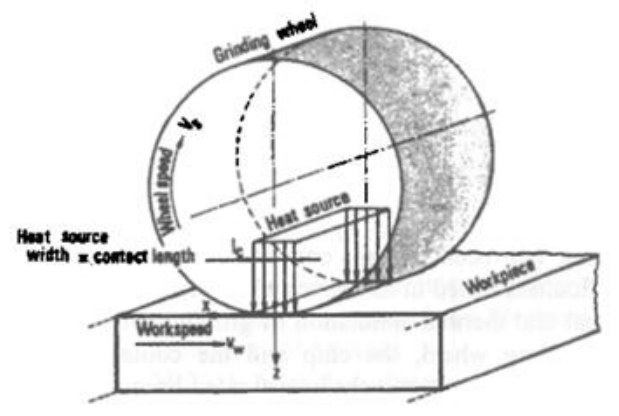

Fig. 2 Jager model

For the experiment they use six aluminium oxide grinding wheels of same diameter and two depths of cut were used while the working speed and wheel speed is constant. But the calculate temperature based on kinematical and geometrical parameters and they had not used any temperature measurement device like thermocouple.

S.Saedodin, et al. In this paper, the melting of a semi-infinite body as a result of moving laser beam has been studied. Because the Fourier heat transfer equation at short times and large dimensions does not have sufficient accuracy; a non-Fourier form of heat transfer equation has been used. The enthalpy technique, used for the solution of phase change problems, has been used in an explicit finite volume form for the hyperbolic heat transfer equation. This technique has been used to calculate the transient temperature distribution in the semi-infinite body and the growth rate of the melt pool. Temporal variation of laser beam intensity in two cases of continuous and pulsatile heat flux has been considered. In order to validate the numerical results, comparisons were made with experimental data. It was reported that the numerical analysis is in good agreement with the experimental data, for low beam intensity. However, in the wake of the reaching to the vaporization threshold, the numerical analysis and experimental data are becoming further from each other. Finally they conclude that, the hyperbolic heat conduction model is suitable for short times and large domains and it can reach to the accurate results. In practical applications such as electric discharge machining (EDM), the pulsatile model has a better effectiveness than Fourier and continuous hyperbolic models.

Babalova et al. The article is focused to the methodology of computer modeling of laser cutting process on stainless steel plate. Computer modeling of laser cutting process is oriented on gradation of models from simple thermal model to combined thermal-fluid analysis. Energy source geometry and beam penetration influence on temperature field in the cutting area are documented by results from the solid model in ANSYS and SYSWELD code. They did this process by three ways-Experimental, ANSYS and SYSWELD. ANSYS is used for creation of 2D model of laser cutting process with shell element. Two dimensional FULL, AIR, and KILLING shell model was done in ANSYS and 3D full and killing solid model was done in SYSWELD. For the heat source distribution they use Gaussian heat source model. They compare these three models and the results showed temperature difference between FULL, AIR and KILLING model.

N.Rajamanickam, et al. For estimating magnitude of welding residual stresses and their nature of distribution along with thermal history, a three dimensional non-linear thermo-mechanical finite element (NLTMFE) model using ANSYS package was developed for butt welded aluminium alloy 2014-T6. Heat source in friction stir welding is considered to be friction between tool shoulder, tool pin and workpiece surface. Local heat generated (dQ) between shoulder and workpiece surface on an elemental area from a distance $(r)$ calculated as

$\mathrm{dQ}=2 \pi \mu \mathrm{F}_{\mathrm{n}} \mathrm{r}^{2} \omega \mathrm{dr}$

where $\mu(0.3-0.5)$ is coefficient of friction varies with temperature, $F_{n}$ is the axial load normal to shoulder and $\omega$ is the angular velocity of shoulder.

Thermal modeling is found useful to predict temperature near tool shoulder. For three welding cases, predicated temperatures match with experiment 
data. Difference of maximum temperature at the same location (weld center) is less than $120 \mathrm{~K}$. Maximum temperature from simulation for all three cases is less than melting point of material. Using NLTMFE model, stress fields in welded plates are simulated to find nature of distribution.

N.Siva Shanmugam, et al. In this study finite element method (FEM) is adopted for predicting the bend geometry in laser welding of $1.6 \mathrm{~mm}$ thick AISI304 stainless steel sheets. Effect of latent heat of fusion and the convective and radiative boundary conditions are included in this model. The heat input is 3D conical Gaussian heat source. The finite element code SYSWELD, along with few FORTRAN subroutines, is employed to obtain the numerical results.

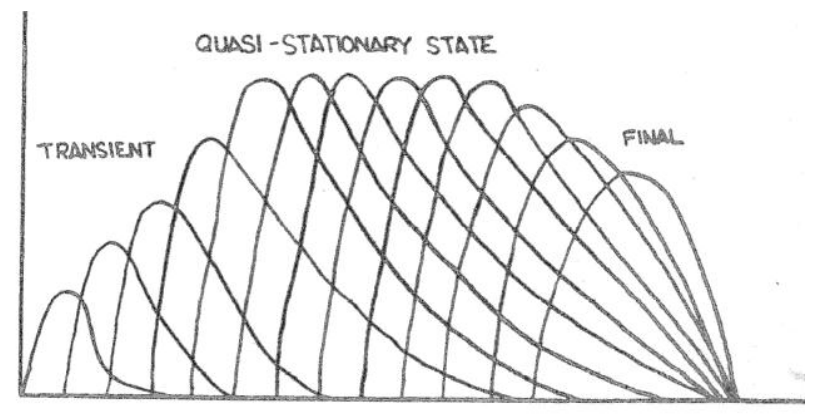

TIME

Fig.3 Stages in heating

They state that heating in laser welding process involves three stages.

- Stationary/Initiation stage- A transient stage at which temperature around the heat source is rising.

- Quasi-Stationary stage- Temperature distribution is stationary in which the co-ordinate system moving with laser source.

- $\quad$ Ending stage- A second transient stage at which temperature profile is decreases after heat source is extinguished.

It is noticed that lot of research is in progress that aims at defining a reliable heat source for the welding processes. Knowledge of heat source from the welding source of the base material is essential for determination of molten pool or bead shape and subsequent solidification pattern.

N.T.Nguyen, et al. In this study, analytical solutions for the transient temperature field of a semi-infinite body subjected to 3-D power density moving heat sources (such as semi-ellipsoidal and double ellipsoidal heat sources) were found and experimentally validated. Also, it was shown the analytical solution obtained for double ellipsoidal heat source was a general one that can be reduced to semi-ellipsoidal, semi-sphere, 2-D Gaussian-distributed heat source and the classical instant point heat source. They studied the effect of heat source parameters on the peak temperature distribution and on the predicted weld pool geometry. A computer program is written in FORTRAN 77 to facilitate the integral calculation and to allow for rapid calculation of transient temperature as well as maximum temperature of any point of interest.

They also check their results with experiment and very good agreement between the predicted transient temperatures and the measured ones at various points in bead-on-plate specimens has been obtained. The predicted geometry of the weld pool is also in good agreement with the measured one. Since many simplified assumptions are made for the development of analytical solution hence this model fails to predict the complex shape of weld pool in the transversal cross section. But there is good agreement between the calculated and measured temperature data indeed shows the credibility of the newly found solution and its potential application for various simulation purposes, such as thermal stress analysis, residual stress calculations and microstructure modeling.

M.Afzaal Malik, et al. The study describes a combination of numerical simulation and experimental validation to study the temperature distribution and prediction of fusion zone (FZ) and heat affected zone (HAZ) in gas tungsten arc welding of low carbon steel. Welding experiments are carried out on weld coupons of $3 \mathrm{~mm}$ thick sheet metals of $150 \times 50 \mathrm{~mm}$ with a 2 mm square gap between them as shown in Fig. 4. Simulation of the entire process is accomplished by using authors written subroutines in ANSYS 10.0, general purpose finite element software. They represents research work on transient non-linear thermal simulation of plate butt joint by arc welding processes using FE heat transfer analysis. A heat source model is presented based on the Goldak's method and MATLAB scripts are developed in order to calculate the heat flux distributions and define a moving heat source into simulation

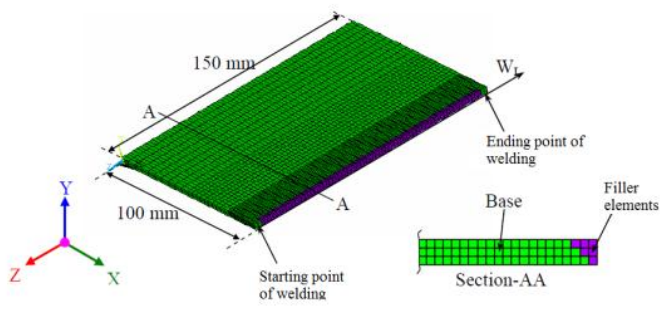

Fig. 4 3D FE model used

In this study, the welded plates and filler material is modeled as solid body. The most widely acceptable double ellipsoidal heat source model, presented by Goldak is used to present the heat generated by the welding torch. The model gives the Gaussian distribution and has excellent features of power and density distribution control in the weld pool and HAZ The variation of the parameters changes the heat source distributions and magnitude, therefore influences the input of heat flux applied in the weld regions. Consequently it affects the transient temperature distributions in the fusion zone, heat affected zone and peak temperatures in the welded 
plates. For that purpose three different set of heat source parameters are used and their effects on the evolution of temperature gradients, fusion and heat affected zones during the arc welding phenomenon are investigated. Different set of GOLDAK's double ellipsoidal heat source parameters are also used.

The induction of carefully measured experimental data into the numerical simulations, showing close corelation between the simulated and experimental investigations in the present study presents the significance of the numerical techniques developed for the precise prediction of transient thermal response of the weldments for the large scale industrial usage in nuclear, aeronautical, aerospace and marine engineering domains.

Han Guo Ming, et al. They propeses the distribution of the temperature field in laser welding, a stinless steel 304 sheet was dynamically simulated bu the FEA software ANSYS.

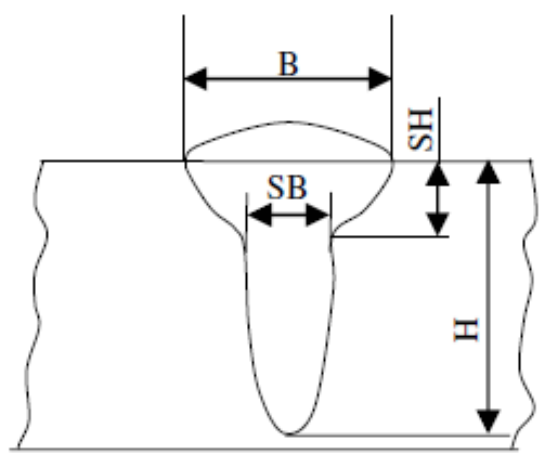

Fig. 5Heat source model used for laser welding

Based on the characteristics of laser welding they proposed heat source model as shown in Fig 5. A travel heat source combined with the body loads was designed by analysing both the temperature relativity of the thermal physical parameters of material and latent heat of fusion and the effect of convection radiation on temperature field.

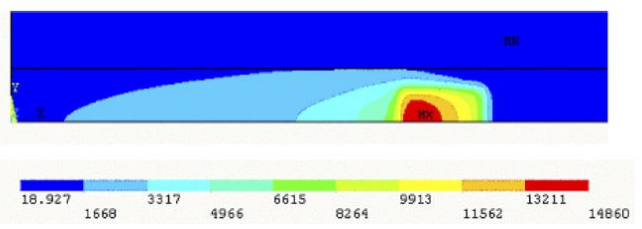

Fig.6 Frontal view of temperature field distribution at $0.30 \mathrm{sec}$

The distribution of temperature field after 0.30 seconds are shown in Fig. 6. The transition element modeling was adopted to take care of highly nonlinear laser welding process. Through the calculation they showed that the simulated results of weld shapewere accordance with the experimental results.

\section{Conclusions}

A major objective in a moving heat source analysis is to determine the temperature field in a medium resulting from conditions imposed on its boundaries. The temperature distribution which represents how temperature varies with position in the medium. Once this distribution is known, the conduction heat flux at any point in the medium or on its surface may be computed from Fourier's Law. Other important quantities of interest may also be determined. For a solid, knowledge of the temperature distribution could be used to discover structural integrity through determination of thermal stresses, expansion, and deflections. The temperature distribution could also be used to optimize the thickness of an insulating material or to determine the compatibility of special coatings or adhesives used with the material.

One can define a differential control volume, identify the relevant energy transfer processes, and introduce the appropriate rate equations to analyze moving heat source problems. The result is a differential equation whose solution, for prescribed boundary conditions, provides the temperature distribution in the medium.

\section{References}

D. Rosenthal,(1946), The theory of moving source of heat and its application to metal transfer, Trans. ASME, vol.43 no.11.

J. Goldak, A. Chakravarti and M. Bibby, (1984): A New Element Model for Welding Heat Source. Metallurgical Transactions B, vol. 15B, pp 299-305.

Bianco N. Manca O. Nardini S. Tamburrino S.(2006), Transient heat conduction in solids irradiated by a moving heat source, Proceedings of COMSOL Users Conference, Milano.

A.G. Mamalis, J. Kundrák, A. Markopoulos, (2003), Numerical simulation for the determination of the temperature fields and the heat affected zones in grinding, Production Systems and Information Engineering, Vol. 1, pp. 3-16.

Y.S. Muzychka and M.M. Yovanovich, (2001), Thermal Resistance Models for Non-Circular Moving Heat Sources on a Half Space, ASME Journal of Heat Transfer, Vol. 123, pp. 624-632.

Babalová, E.; Taraba, B. \& Duehring, S. (2012), Computer modeling methodology for laser cutting process supported with experiment on stainless steel plate International DAAAM Baltic Conference INDUSTRIAL ENGINEERING, Tallinn, Estonia.

Rajamanickam N, Balusamy V, Thyla P R and Hari Vignesh G, (2009), Numerical Simulation of Thermal History and Residual Stresses in Friction Stir Welding of Al 2014 - T6, Journal of Scientific and Industrial Research, Vol.68, pp.192-198.

Shanmugam N. Siva, G. Buvanashekaran, K. Sankaranarayanasamy, S. Ramesh Kumar (2010), A transient finite element simulation of the temperature and bead profiles of T-joint laser welds, Materials \& design 31, P. 4528-4542.

N. T. Nguyen, A. Ohta, K. Matsuoka, N. Suzuki, and Y. Maeda, (1999), Analytical solutions for transient temperature of semi-infinite body subjected to 3-D moving heat sources, Welding Research, vol. 78, no. 8, pp. 265-S-274-S.

M. Afzaal Malik, M. Ejaz Qureshi and Naeem Ullah Dar, (2007) Numerical Simulation of Arc Welding: Investigation of Various Process and Hea Source Parameters, Available in the proceedings of Conference on Failure of Engineering Materials \& Structures (FEMS 2007), Taxila, Pakistan.

Brockmann, R. Dickmann, K. Geshev, P. Matthes, K.J. (2003), Calculation of temperature field in a thin moving sheet heated with laser beam. Int Journal of Heat and Mass Transfer, 46,717-723.

Qasim M. Doos, Dr. Muhsim Jabir Jweeg, Sarmad Dhia Ridha. (2008), Analysis of friction stir welds. Part-I: Transient thermal simulation using moving het source. Regional conference of Eng. Sci. NUCEJ spatial issue vol. 11, No.3 pp 429-437.

D. Gery, H. Long, P. Maropoulos, (2005), Effects of welding speed energy input and heat source distribution on temperature variations in butt join welding, Journal of Materials Processing Technology 167 393-401.

Ivanović Ivana B., Sedmak Aleksandar S., Miloš Marko V., Živković Aleksandar B., Lazić Mirjana M. (2011), Numerical study of transient three-dimensional heat conduction problem with a moving heat source. THERMAL SCIENCE, 15(1):257-266.

Lazić, V.N., Sedmak, A.S., Živković, M.M., Aleksandrović, S.M., Čukić, R.D., Jovičić, R.D., Ivanović, I.B. (2010), Theoretical-experimental determining of cooling time (t8/5) in hard facing of steels for forging dies. Thermal Science, vol. 14 br. 1 , str. $235-246$

P. Duranton, J. Decaux, V. Robin, P. Gilles and J. M. Bergheu, (2004), 3D modelling of multipass welding of a $316 \mathrm{~L}$ stainless steel pipe, Journal of Material Processing Technology 153-154 457-463.

Hang Guo Ming, Zhao Jian, Li Jian Qang, (2007). Dynamic simulation of the temperature field of stainless steel laser welding, Material and Design 28 240-245. 